\title{
Mineração
}

\section{O processo eletroquímico como alternativa para o tratamento de efluentes cianídricos}

\author{
Achilles Junqueira Bourdot Dutra \\ Engenheiro Metalúrgico, D.Sc., Professor Adjunto, COPPE/UFRJ-PEMM. E-mail: adutra@metalmat.ufr.br \\ Luiz Gonzaga Santos Sobral \\ Engenheiro Químico,Ph.D., Centro de Tecnologia Mineral -CETEM.E-mail: Isobral@cetem.gov.br \\ Flávio de Almeida Lemos \\ Engenheiro Químico,M.Sc., COPPE/UFRJ-PEMM.E-mail: lemos@metalmat.ufrj.br \\ Fábio Henrique Silva dos Santos \\ Químico, Mestrando, COPPE/UFRJ-PEMM.E-mail: fsantos@metalmat.ufrj.br
}

\section{Resumo}

Embora o cianeto seja um reagente amplamente empregado na lixiviação de metais preciosos e no acabamento superficial de certos metais, sua elevada toxicidade faz com que o uso de tecnologias eficientes no tratamento dos efluentes desses processos seja primordial. Existem diversas técnicas disponíveis para o tratamento de efluentes industriais contendo cianeto, contudo os métodos mais empregados baseiam-se na oxidação química do cianeto a uma espécie menos tóxica, o cianato, que se hidrolisa produzindo os íons amônio e bicarbonato, além de uma lama contendo hidróxidos de metais pesados, quando presentes no efluente. Entre as técnicas mais recentes para o tratamento de efluentes contendo cianeto, a oxidação eletroquímica apresenta algumas vantagens, como a possibilidade de recuperação dos metais complexados pelo cianeto, além de não necessitar de reagentes químicos adicionais, sendo perfeitamente capaz de atender às exigências dos órgãos ambientais. Esse trabalho apresenta uma comparação entre os diversos processos de tratamento de efluentes contendo cianeto, com ênfase no processo eletroquímico, e também uma revisão sobre estado atual dessa tecnologia.

Palavras-chave: Cianeto, Efluente, Oxidação, Meio ambiente.

\begin{abstract}
Although cyanide is widely employed for leaching precious metals and electroplating certain metals, its high toxicity demands efficient technologies to treat the wastewater generated by these processes. There are a number of available techniques for the treatment of industrial aqueous cyanide-bearing effluents. However, the most employed method is based on its chemical oxidation to the less toxic cyanate which is hydrolyzed to ammonium and bicarbonate ions. The heavy metal complexes, when present in the effluent, will form a sludge composed of hydroxides. Among the most recent techniques for the treatment of effluents containing cyanide, electrochemical oxidation presents some advantages, such as: possible metal recovery from the cyano-complexes, no need for further chemicals additions, compliance to environmental agencies directives. This paper presents a comparison among several processes for treating the aforementioned effluents, with emphasis on the electrochemical process, which is briefly reviewed.
\end{abstract}

Keywords: Cyanide, Effluent, Oxidation, Environment.

Artigo recebido em 31/07/2002 e aprovado em 17/10/2002 


\section{Introdução}

Desde o século XIX que o homem tem desenvolvido e aperfeiçoado várias técnicas e atividades industriais, visando a produzir mais em quantidade e, se possível, em qualidade. Dessa produção em massa, resultam, quase sempre, efluentes, ou seja, descartes líquidos que, muitas vezes, são lançados inadvertidamente nos corpos receptores sem tratamento prévio, impactando o ecossistema em um dos seus constituintes mais valiosos: á água. Isto acarreta prejuízos econômicos e ambientais incalculáveis. A Organização das Nações Unidas já prevê que, nesse século, a água será o bem mais precioso do mundo.

O cianeto, por sua vez, é um dos principais agentes poluidores desses recursos hídricos. As maiores fontes de agentes poluidores são os descartes de processos de mineração, usinas siderúrgicas, indústrias químicas de compostos orgânicos e estações de tratamento de água (Harper \& Goldhaber, 1997). Outras fontes de cianeto incluem a exaustão de veículos, rejeitos de certas indústrias químicas, queima de lixo em aterros sanitários e uso de pesticidas contendo cianeto (Harper \& Goldhaber, 1997).

Estima-se que anualmente são manufaturados mundialmente cerca de 2,6 milhões de toneladas de produtos de cianeto. Aproximadamente $20 \%$ da produção mundial de cianeto, ou seja, cerca de 0,6 milhões de toneladas, é utilizada na mineração. A maior parte desta, cerca de $95 \%$, é empregada na lixiviação de ouro e prata. Os 5\% restantes são utilizados em grande parte como agente depressor em operações de flotação, visando à separação de metais como cobre, chumbo, molibdênio e zinco. Os $80 \%$ restantes da produção mundial de cianeto são utilizados nas indústrias de corantes, quelantes, tintas e pigmentos, indústrias de plásticos, fibras e detergentes, na produção de fármacos, pesticidas e herbicidas, na preparação de alimentos e nas indústrias metalúrgicas para processos de acabamento superficial de metais (Yong, 2001).

Nos últimos trinta anos, ocorreu, em média, um vazamento em bacias de con- tenção por ano, sendo que um terço desses acidentes envolveram cianeto. Tal fato faz com que a opinião pública mundial tenha uma imagem negativa das empresas de mineração, no que concerne aos impactos ambientais por elas causados (Mackie, 2001).

Os vazamentos acidentais de soluções contendo cianeto, em rios e cursos d'água, têm produzido uma grande quantidade de mortes na fauna aquática, bem como destruído plantações ribeirinhas.

O maior acidente envolvendo vazamento de cianeto ocorreu em 30 de janeiro de 2000 na Romênia (baía de Maré), que acarretou a morte de 20.000 trutas e contaminou cerca de $320 \mathrm{~km}$ do rio Danúbio (Miller, 2001).

A Tabela 1 apresenta os principais acidentes envolvendo cianeto em minerações ocorridos no mundo nos últimos anos.

No Brasil, podem ser relatados a poluição da baía de Babitonga localiza- da próxima ao pólo industrial da grande Joinville (SC), onde indústrias de galvanoplastia descartaram efluentes com elevados teores de metais pesados e cianeto; o córrego do Jacu (MG), que foi contaminado pela lavra da Serra Luís Soares e a geração de 9,0 toneladas/dia de lama contendo cianeto e metais pesados de cerca de 300 indústrias de galvanoplastia na cidade de Limeira (SP), considerada a capital brasileira da jóia (Aguaonline $n^{\circ} 14,2000$ ).

A falta de tratamento de resíduos contendo cianeto, aliada ao descarte em locais inadequados para receber lixo tóxico e, ainda, à falta de informação da população, é uma das principais causas de intoxicação de seres humanos por cianeto, já tendo, inclusive, causado acidentes fatais, como o ocorrido com uma criança em julho de 2000 no Rio de Janeiro.

O descarte de efluentes deve seguir, obrigatoriamente, os padrões estabelecidos pelo órgão ambiental local. $\mathrm{Na}$

Tabela 1 - Acidentes envolvendo contaminação por cianeto (Miller e Pritsos, 2001).

\begin{tabular}{c|c|l}
\hline Local & \multicolumn{1}{|c}{ Ano } & \multicolumn{1}{c}{ Impacto } \\
\hline Gana & Outubro de 2001 & $\begin{array}{l}\text { Rompimento da bacia de contenção da } \\
\text { South African Company Goldfields Ltd., } \\
\text { contaminando o rio Asuman, causando a } \\
\text { morte de centenas de peixes, crustáceos } \\
\text { e aves. }\end{array}$ \\
\hline Romênia & Janeiro de 2000 & $\begin{array}{l}\text { Mais de 100 m }{ }^{3} \text { de uma mistura, } \\
\text { contendo cianeto e metais pesados, } \\
\text { vazaram de uma mineração de ouro } \\
\text { contaminando o rio Tisza, Romênia, } \\
\text { causando a morte de pelo menos 15 } \\
\text { toneladas de peixe. }\end{array}$ \\
\hline Gana & Junho de 1997 & $\begin{array}{l}\text { Vazamento na mina de ouro Teberebie, } \\
\text { contaminando o rio Angonaben } \\
\text { acarretando na morte de peixes e } \\
\text { destruindo todas as plantações às } \\
\text { margens do rio. }\end{array}$ \\
\hline Guiana & Agosto de 1995 & $\begin{array}{l}\text { Rompimento da bacia de contenção da } \\
\text { Omai Gold Mines Ltd., despejando 2,9 } \\
\text { milhões de toneladas de efluente e } \\
\text { resíduos no rio Omai. }\end{array}$ \\
\hline
\end{tabular}


Tabela 2, encontram-se listados os padrões estabelecidos por alguns órgãos públicos ambientais para o cianeto, $\mathrm{pH}$ do efluente e para o cobre, metal comumente associado aos efluentes contendo cianeto em minerações e galvanoplastias.

\section{Processos de trata- mento de efluentes contendo cianeto}

Os métodos de tratamento de efluentes contendo cianeto podem ser classificados de diversas formas. No presente trabalho, agruparam-se esses processos em duas grandes classes: aqueles que degradam o cianeto (e removem o metal) e aqueles que promovem a reciclagem do cianeto além da remoção do metal da solução. A Tabela 3 apresenta os principais processos.

A escolha do processo mais adequado para o tratamento de um efluente contendo cianeto será função de certos fatores. Entre eles destacam-se (Smith \& Mudder, 1991):

- Concentração e composição do efluente a tratar.

- Qualidade final desejada no despejo e legislação ambiental local.

- Localização da unidade de tratamento, disponibilidade e preços de reagentes e insumos, topografia, área disponível para implantação da unidade, etc.

- Tipo de processo que gerou o efluente (galvanoplastia, eletrorrecuperação, etc).

- Escala de operação da unidade geradora do efluente.

- Custos de capital e de operação da unidade de tratamento.

- Consumo de cianeto.

A Tabela 4 ilustra as vantagens e desvantagens dos principais processos industriais empregados na destruição de cianeto (Parga \& Miller, 2001).

Deve ser mencionado que os agentes oxidantes, como o peróxido de hidrogênio, oxidam o cianeto livre, mas pro- duzem paralelamente lamas contendo hidróxidos de metais pesados, decorrentes da reação com o cianeto complexo, como mostra a reação (1), para o caso do cobre. Já no processo eletroquímico o cianeto livre é oxidado anodicamente, enquanto que o metal pode ser depositado no catodo, com a liberação do cianeto, que poderá tanto ser oxidado como reciclado, dependendo do projeto da célula, como mostram as reações (2) e (3).

$\mathrm{Cu}(\mathrm{CN})_{x}^{y-x}+x \mathrm{H}_{2} \mathrm{O}_{2}+y \mathrm{OH}^{-} \quad \mathrm{Cu}(\mathrm{OH})_{y}+x \mathrm{OCN}^{-}+x \mathrm{H}_{2} \mathrm{O}$

$\mathrm{CN}^{-}+2 \mathrm{OH}^{-} \quad \mathrm{OCN}^{-}+\mathrm{H}_{2} \mathrm{O}+2 e^{-}$

$C u(C N)_{x}^{y-x}+y e^{-} \quad C u+x C N^{-}$

Tabela 2 - Padrões para descarte de efluentes contendo cobre e cianeto.

\begin{tabular}{c|c|c|c}
\hline \multirow{2}{*}{ Órgão Ambiental } & \multicolumn{3}{|c}{ Padrões } \\
\cline { 2 - 4 } & $\begin{array}{c}{[\mathbf{C u}]} \\
\left(\mathrm{mg} \mathrm{dm}^{-3}\right)\end{array}$ & $\begin{array}{c}{[\mathbf{C N}]_{\text {total }}} \\
\left(\mathbf{m g ~ d m}^{-3}\right)\end{array}$ & $\mathbf{p H}$ \\
\hline Conama & 1 & 0,2 & $5-9$ \\
\hline Feema & 0,5 & 0,2 & $5-9$ \\
\hline Cetesb & - & 0,2 & $5-9$ \\
\hline Copam & 0,5 & 0,2 & $6,5-8,5$ \\
\hline
\end{tabular}

Tabela 3 - Processos usuais de tratamento de efluentes cianídricos (Mackie, 2001; Huiatt et al., 1982).

\begin{tabular}{|c|c|c|}
\hline \multicolumn{3}{|c|}{ Degradação de cianetos } \\
\hline $\begin{array}{c}\text { Degradação } \\
\text { natural }\end{array}$ & Processos oxidativos & Outros processos \\
\hline \multirow{2}{*}{ Diluição } & Cloração alcalina & \multirow{6}{*}{$\begin{array}{l}\text { Tratamento biológico } \\
\text { Conversão em formas } \\
\text { menos tóxicas } \\
\text { (tiocianato e ferrocianeto) }\end{array}$} \\
\hline & Cloro & \\
\hline \multirow{2}{*}{ Volatilização } & Hipoclorito & \\
\hline & Geração eletrolítica & \\
\hline \multirow{2}{*}{ Biodegradação } & de cloro (in situ) & \\
\hline & Ozônio & \\
\hline Oxidação & Peróxido de hidrogênio & Processos eletrolíticos \\
\hline $\begin{array}{l}\text { Precipitação de } \\
\text { metais }\end{array}$ & Processo Inco $\left(\mathrm{SO}_{2} / \mathrm{ar}\right)$ & Carvão ativado \\
\hline \multicolumn{3}{|c|}{ Processos de reciclagem de cianeto } \\
\hline \multicolumn{3}{|c|}{ Processo de acidificação, volatilização e reneutralização (AVR). } \\
\hline \multicolumn{3}{|c|}{ Resinas de troca iônica. } \\
\hline \multicolumn{3}{|c|}{ Processo eletrolítico. } \\
\hline \multicolumn{3}{|c|}{ Resina quelante vitrokele ${ }^{\circledR}$. } \\
\hline
\end{tabular}


Tabela 4 - Comparação dos processos de destruição de cianeto.

\begin{tabular}{|c|c|c|}
\hline Agente oxidante & Vantagens & Desvantagens \\
\hline Cloração alcalina & $\begin{array}{l}\text { Tecnologia bem estabelecida. } \\
\text { Cianeto é menos tóxico que cianato e } \\
\text { pode ser oxidado a } \mathrm{CO}_{2} \text { e } \mathrm{N}_{2} \text { em baixos } \\
\text { valores de } \mathrm{pH} \text {. }\end{array}$ & $\begin{array}{l}\text { A reação de cloro com compostos } \\
\text { orgânicos pode gerar organoclorados. } \\
\text { Geração de intermediários tóxicos. } \\
\text { Reage preferencialmente com tiocianato. } \\
\text { Hipoclorito em excesso é tóxico e requer } \\
\text { estocagem especial. }\end{array}$ \\
\hline Peróxido de hidrogênio & $\begin{array}{l}\text { Operação simples. } \\
\text { Decomposição do excesso de reagente } \\
\text { em } \mathrm{H}_{2} \mathrm{O} \text { e } \mathrm{O}_{2} \\
\text { Não reage com tiocianato. }\end{array}$ & $\begin{array}{l}\text { Custo do reagente. } \\
\text { A precipitação de ferrocianetos com } \\
\text { cobre deve ser feita separadamente. }\end{array}$ \\
\hline $\mathrm{SO}_{2} / \mathrm{Ar}(\mathrm{INCO})$ & $\begin{array}{l}\text { Baixo custo dos reagentes } \\
\text { Tratamento de soluções aquosas e } \\
\text { polpas. }\end{array}$ & $\begin{array}{l}\text { Pagamento de royalty. } \\
\text { Adição de sulfatos na água tratada. } \\
\text { Se realizar precipitação de ferrocianetos } \\
\text { com cobre, este deve ser separado dos } \\
\text { demais precipitados. }\end{array}$ \\
\hline Ozônio & Possível regenerar parte do cianeto. & $\begin{array}{l}\text { Custo do reagente. } \\
\text { Custo dos equipamentos. }\end{array}$ \\
\hline $\begin{array}{l}\text { Oxidação } \\
\text { eletroquímica }\end{array}$ & $\begin{array}{l}\text { Equipamentos simples. } \\
\text { Pode tratar soluções diluídas e } \\
\text { concentradas. } \\
\text { Controle do processo. } \\
\text { Recuperação do metal. } \\
\text { Não gerar intermediários tóxicos. }\end{array}$ & Pode requerer tratamentos adicionais. \\
\hline
\end{tabular}

\section{Oxidação eletroquí- mica do cianeto}

A oxidação eletroquímica do cianeto foi primeiramente apresentada por Clevenger e Hall em 1913 e apresentou-se como uma alternativa viável para tratamento de efluentes contendo cianetos devido ao fato de não gerar intermediários tóxicos, permitindo tanto a reciclagem como a decomposição do cianeto (Smith \& Mudder, 1991).

Quando a concentração de cianeto na solução é reduzida abaixo de $100 \mathrm{mgdm}^{-3}$, os métodos eletroquímicos não são tão eficientes devido à limitação na transferência de massa e à baixa efici- ência de corrente. O problema de limitação de transferência de massa em eletrólitos muito diluídos, tais como os efluentes cianídricos, pode ser resolvido pelo uso de eletrodos porosos. Tais eletrodos promovem, não apenas uma alta taxa de transferência de massa, mas também possuem uma elevada área superficial num volume compacto (Hofseth \& Chapman, 1999).

Dois parâmetros são fundamentais para a viabilidade econômica de um reator eletroquímico utilizado na destruição de cianeto, quando comparado com outros sistemas comerciais: a concentração de cianeto do efluente e a eficiência de corrente do sistema reacional. Reatores que não reduzem a concentração em níveis inferiores a $0,1 \mathrm{mg} \mathrm{dm}^{-3}$ podem necessitar de tratamentos adicionais para o descarte de efluentes dentro dos padrões ambientais exigidos. A eficiência de corrente, por sua vez, não deverá ser inferior a $10 \%$ para que o processo eletroquímico possa competir com outras tecnologias de oxidação de cianetos (Martins, 2001).

Na Tabela 5, são apresentados alguns dados de trabalhos envolvendo a oxidação eletroquímica de cianeto, empregando-se diferentes tipos de anodos e condições operacionais.

A rota de oxidação eletroquímica do cianeto é potencialmente atrativa, uma 
Tabela 5 - Dados sobre a oxidação eletroquímica do cianeto.

\begin{tabular}{|c|c|c|}
\hline Pesquisadores & Tipo de Anodo & Observações \\
\hline Fockedey et al. (2001) & $\begin{array}{l}\text { Feltro de carbono revestido } \\
\qquad \mathrm{com} \mathrm{CoO}_{2}\end{array}$ & $\begin{array}{l}\text { Cobalto exerce função catalítica. } \\
\text { Densidade de corrente: } 400 \mathrm{~A} \mathrm{~m}^{-2} \text {. } \\
\text { Consumo energia esp. } 9,7 \mathrm{kWh} \mathrm{kg}^{-1} \mathrm{CN} \text {. } \\
\text { Concentração final } \mathrm{CN}: 5,0 \mathrm{mg} \mathrm{dm}^{-3} \text {. }\end{array}$ \\
\hline Zhou e Chin (1993) & - & $\begin{array}{l}\text { Consumo energia esp. } 340 \mathrm{kWh} \mathrm{kg}^{-1} \mathrm{CN} \text {. } \\
\text { Concentração final } \mathrm{CN}^{-}: 10 \mathrm{mg} \mathrm{dm}^{-3}\end{array}$ \\
\hline Tissot e Fragnière (1994) & $\begin{array}{l}\text { Carbono vítreo reticulado de } \\
30 \text { ppi revestido com } \mathrm{PbO}_{2} \text {. }\end{array}$ & $\begin{array}{l}\text { Densidade de corrente: } 4,25 \mathrm{~A} \mathrm{~m}^{-2} \text {. } \\
\text { Vazão eletrólito: } 6,6 \times 10^{-6} \mathrm{~m}^{3} \mathrm{~h}^{-1} \text {. } \\
\text { Concentração final } \mathrm{CN}^{-}: 5,0 \mathrm{mg} \mathrm{dm}^{-3} \text {. } \\
\text { De } 80-90 \% \text { do } \mathrm{CN}^{-} \text {oxidado a } \mathrm{HCO}_{3}^{-}\end{array}$ \\
\hline Hosefh e Chapman (1999) & $\begin{array}{l}\text { Carbono vítreo reticulado de } \\
100 \text { ppi (pores per inch). }\end{array}$ & $\begin{array}{l}\text { Reação catalisada pela adição de cobre. } \\
\text { Potencial catódico: } 1,1 \mathrm{~V} \text {. } \\
\text { Concentração final } \mathrm{CN}^{-}: 0,78 \mathrm{mg} \mathrm{dm}^{-3} \\
\text { Eficiência de corrente: } 5,0 \% \text {. }\end{array}$ \\
\hline Szpyrkowicz et al.(2000) & Placa de Ti/Pt & $\begin{array}{l}\text { Densidade de corrente: } 2,0 \mathrm{~A} \mathrm{~m}^{-2} \text {. } \\
\text { Concentração final } \mathrm{CN}^{-}: 7,9 \mathrm{mg} \mathrm{dm}^{-3} \text {. }\end{array}$ \\
\hline Sandord e Martins (2001) & Feltro de carbono & $\begin{array}{l}\text { Estudou efeito da condutividade do eletrólito na } \\
\text { oxidação de } \mathrm{CN}^{-} \text {. } \\
\text { Corrente de trabalho: } 15 \mathrm{~mA} \text {. } \\
\text { Velocidade do eletrólito: } 0,0232 \mathrm{~cm} \mathrm{~s}^{-1} \\
\text { Concentração final } \mathrm{CN}^{-}: 0,002 \mathrm{mg} \mathrm{dm}^{-3}\end{array}$ \\
\hline Stavard e Lierde (2001) & Titânio revestido com $\mathrm{Co}_{3} \mathrm{O}_{4}$. & $\begin{array}{l}\text { Densidade de corrente: } 100 \mathrm{~A} \mathrm{~m}^{-2} \text {. } \\
\text { Concentração final } \mathrm{CN}^{-}: 0,2 \mathrm{mg} \mathrm{dm^{-3 }} \text {. } \\
\text { Eficiência de corrente: } 28,5 \% \text {. }\end{array}$ \\
\hline Fugivara et. al. (1996) & $\begin{array}{l}\text { Titânio revestido com } \mathrm{SnO}_{2} \\
\text { dopado com } \mathrm{Sb}\end{array}$ & 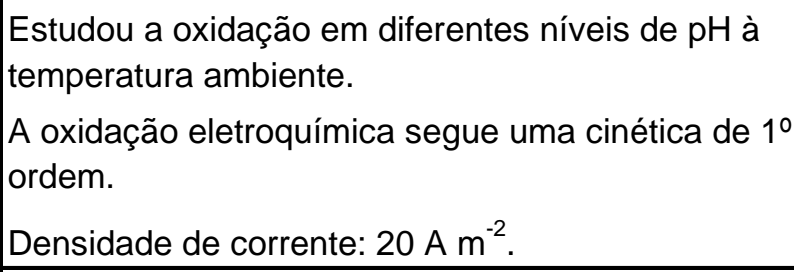 \\
\hline Cheng et. al. (2002) & Ti platinizado & $\begin{array}{l}\text { Potencial anódico: } 0,90 \mathrm{~V} \text {. } \\
\text { Concentração final CN: } 85 \mathrm{mg} \mathrm{dm}{ }^{-3} \text {. } \\
\text { Eficiência de corrente: } 63,0 \% \text {. }\end{array}$ \\
\hline Lanza e Bertazzoli (2002) & $\begin{array}{l}\text { Titânio revestido com } \\
\mathrm{TiO}_{2} / \mathrm{RuO}_{2} \text { e } \mathrm{Ta}_{2} \mathrm{O}_{5} / \mathrm{lrO} \text {. }\end{array}$ & $\begin{array}{l}\text { Estudos de voltametria linear e eletrólise em } \\
\text { potencial constante. }\end{array}$ \\
\hline Lemos (2002) & Chapa de grafita & $\begin{array}{l}\text { Vazão eletrólito: } 25,8 \mathrm{dm}^{3} \mathrm{~h}^{-1} . \\
\text { Concentração final } \mathrm{CN}_{\text {Total }}: 3,63 \mathrm{mg} \mathrm{dm}^{-3} \text {. } \\
\text { Consumo energia esp. } 301 \mathrm{kWh} \mathrm{kg}^{-1} \mathrm{CN} \text {. }\end{array}$ \\
\hline
\end{tabular}


vez que não requer locais para estocagens de agentes oxidantes, tais como cloro, hipoclorito ou peróxido de hidrogênio e oferece a possibilidade de se recuperar o metal dissolvido em uma de suas formas mais valiosas, isto é, um sólido metálico puro, o que pode reduzir os custos operacionais da etapa de tratamento dos efluentes.

Outros processos competitivos (precipitação de íons metálicos como hidróxidos, extração por solvente e troca iônica) conduzem à precipitação de um sal ou a um outro íon metálico em concentração mais elevada, enquanto a cementação gera um metal com elevado grau de impureza.

A expansão da tecnologia eletroquímica requer o desenvolvimento de anodos dimensionalmente estáveis, com alto rendimento elétrico na destruição do cianeto. Uma larga gama de anodos têm sido estudados, como, por exemplo: platina, ligas de titânio, níquel, grafita, carbono vítreo reticulado revestido com óxido de chumbo e titânio revestido com óxido de cobalto.

\section{Conclusões}

A estocagem de grandes quantidades de efluentes não tratados em bacias de contenção mostra ser um procedimento inadequado do ponto de vista ambiental, visto que pode ocasionar acidentes de grandes proporções.

A literatura fornece diversas opções para o tratamento de efluentes cianídricos, que devem ser utilizados de acordo com as particularidades dos processos em questão.
O processo de oxidação eletroquímica de cianeto demonstra ser uma alternativa viável para tratamento de efluentes, uma vez que requer equipamentos simples e de fácil controle operacional, se adaptando, assim, a pequenas unidades de mineração e galvanoplastia. Esse processo apresenta a vantagem de não gerar intermediários tóxicos, lamas com elevados teores de metais pesados e cianeto e recuperando o metal contido na solução de uma forma pura no catodo, além de, em alguns casos, dispensar tratamentos adicionais.

\section{Referências Bibliográficas}

AGUAONLINE $n^{\circ} 14$ - 05/07 a 11//07/2000. CHENG, S.C. et al. The electrochemical oxidation of alkaline copper cyanide solutions. Electrochim. Acta (in press), 2002.

FOCKEDEY, E., et al. Electrochemical destruction of free cyanide on a cobalt oxide doped electrode. Cyanide Social, Industrial and Economic Aspects. In: YOUNG, C. (ed.). TMS, 2001. p.401-411.

FUGIVARA, C.S, et al. Electrochemical Decomposition of cyanides on tin dioxide electrodes in alkaline media. Analyst, v.121, n. 4 p. 541-545, 1996.

HINE F. et al. On the oxidation of cyanide solutions with lead dioxide coated anode. Electrochim. Acta, v. 31 n.11, p.13891395, 1986.

HOFSETH, C. S., CHAPMAN, W. Electrochemical destruction of dilute cyanide by copper-catalyzed oxidation in a flow-through porous electrode. $\boldsymbol{J}$. Electrochem. Soc., v.146 n.1, p.199-207, 1999.

HUIATT, L. J. et al. Control and treatment of cyanide mining wastes. In: Proceedings of a Workshop on Cyanide from Mineral Processing. Chapter 2, p.2-5, Salt Lake City, Utah, 1982.

LANZA, M.R.V. and BERTAZZOLI, R. Selection of commercial anode oxide coating for electro-oxidation of cyanide. Journal of the Brazilian Chemical Society., v.13, n.3 p.345-351, 2002.
LEMOS, F.A. Remoção eletrolítica de cobre de efluentes aquosos com reciclagem de cianeto. COPPE/UFRJ, 2002. (Dissertação de mestrado).

MACKIE A.D. Cyanide leaching, interrupted. In: Cyanide: Social, Industrial and Economic Aspects. New Orleans, 2001. p.51-60.

MARTINS, G. P. Oxidation of cyanide in an electrochemical porous-electrode flowreactor. In: Cyanide Social, Industrial and Economic Aspects. In: YOUNG, C. (ed.) TMS, 2001. p.386-401.

MILLER, G. C., PRITSOS, C. A. Unresolved problems with the use of cyanide in open pit precious metals mining. In: Cyanide: Social, Industrial and Economic Aspects. New Orleans, 2001. p.73-81.

PARGA, J.R., MILLER, J. D. Cyanide recovery/ destruction using air sparged hydrocyclone technology. In: Cyanide: Social, Industrial and Economic Aspects. New Orleans, 2001. p. 363-377.

SANFORD, R. P., MARTINS, G. P. Oxidation of cyanide in an electrochemical porouselectrode flow-reactor In: Cyanide Social, Industrial and Economic Aspects. In: YOUNG, C. (ed.), TMS, 2001. p.386-401.

SMITH, A., MUDDER, T. The chemistry and treatment of cyanidation wastes. Chapters 1, 2, 5 and 6. London, England: Mining Journal Books Limited, 1991.

STAVARD, A., LIERDE A.V. Electro-oxidation of cyanide on cobalt oxide anodes. J. Appl. Electrochem., v. 31 n.4 p.469-474, 2001.

SZPYRKOWICZ, L. et al. Copper electrodeposition and oxidation of complex cyanide from wastewater in a electrochemical reactor with a $\mathrm{Ti} / \mathrm{Pt}$ anode. Ind. Eng. Chem. Res., 39, p.21322139, 2000.

TISSOT, P., FRAGNIÈRE, M. Anodic oxidation of cyanide on a reticulated threedimensional electrode. J. Appl. Electrochem., v. 24, p. 509-512, 1994.

HARPER, C., GOLDHABER, S. Toxicological profile for cyanide. U.S. Dept. of Health and Human Services. Atlanta, Sept. 1997.

YONG, C.A. CYANIDE: just the facts. In: Cyanide: Social, Industrial and Economic Aspects. New Orleans, 2001. p. 97-113.

ZHOU, C.D., CHIN, D.T. Copper recovery and cyanide destruction with a plating barrel cathode and a packed-bed anode Plating and Surface Finishing. p. 69-78, June 1993.

\section{Fale conosco www.rem.com.br remjorio@ouropreto.com.br}

